\title{
Natural history of AIDS related sclerosing cholangitis: a study of 20 cases
}

\author{
A Forbes, C Blanshard, B Gazzard
}

\begin{abstract}
A case control study of AIDS related sclerosing cholangitis indicates that it has no overall influence on prognosis, but is responsible for a striking reversal of the usual inverse correlation of age and survival in HIV infection. Pain, the principal symptom, was controlled in surviving patients with analgesics alone. Twenty consecutive patients with AIDS related sclerosing cholangitis, defined from at least two characteristic lesions at endoscopic retrograde cholangiopancreatography, were followed for a minimum of $\mathbf{1 0}$ months or until death. Median age was 33.5 years (range 2750). All had abdominal pain; 11 had diarrhoea. Alkaline phosphatase was $>2 \times$ normal in 13 , but the bilirubin was raised in only three. The median CD4 was $0.024 \times 10^{\circ} / 1(0.005-0.341)$. Thirteen had cryptosporidiosis, six had active cytomegalovirus, five had no gastrointestinal pathogen. Three patients are alive without AIDS related sclerosing cholangitis symptoms at 10, 11, and 21 months. Seventeen have died at median 7 (1-23) months. Cytomegalovirus therapy had no apparent influence. The initial CD4 was $<0.11$ in all those dying within six months, but correlation of CD4 with prognosis was otherwise poor. Controls, matched for age, CD4, and opportunistic infections had virtually identical overall outcome (median survival 7.5 months) and the expected worse prognosis with increasing age. Increasing age, however, appeared protective in AIDS related sclerosing cholangitis $(r=+0.6 ; p<0.05)$ : this is not explained by disproportionate degrees of immunosuppression, nor by opportunistic infections.

(Gut 1993; 34: 116-121)
\end{abstract}

Investigation of HIV infected patients with abdominal pain has led to the recognition of a condition now widely termed AIDS related sclerosing cholangitis. ${ }^{12}$ Typically patients have right upper quadrant or epigastric pain and obstructive liver function tests. Ultrasound scanning may show thickening of the bile ducts and less often provides evidence of bile duct stricturing and/or dilatation..$^{13}$ Diagnosis is made from features on direct cholangiography indistinguishable from those of primary sclerosing cholangitis, but there is no link with inflammatory bowel disease. In some cases AIDS related sclerosing cholangitis and cryptosporidial or cytomeglaovirus enteritis coexist, ${ }^{12+}$ but whether this is a causal relationship remains unresolved. The natural history of AIDS related sclerosing cholangitis has not been clearly documented, and it is not known whether its presence has an independent influence on the prognosis of the affected patient. Twenty consecutive patients with AIDS related sclerosing cholangitis have therefore been studied to address these questions.

\section{Methods}

PATIENTS

All patients presenting to the Westminster AIDS Unit with abdominal pain severe enough to require admission and with a life expectancy considered to be of three months or more are investigated in accordance with a previously described protocol. ${ }^{5}$ This usually implies upper gastrointestinal endoscopy, ultrasound examination, and investigation for enteric pathogens. Unexplained pain, abnormal liver function tests or biliary abnormality at ultrasound lead to endoscopic retrograde cholangiopancreatography, and thereby a potential diagnosis of AIDS related sclerosing cholangitis. The present report concerns consecutive patients with a firm diagnosis of AIDS related sclerosing cholangitis made between March 1987 and May 1991: all were investigated prospectively to the agreed standard protocol.

Diagnosis of AIDS related sclerosing cholangitis for the purpose of this study required HIV seropositivity by two separate methods, and changes on direct cholangiography that were compatible with diagnostic criteria for primary sclerosing cholangitis. ${ }^{6}$ Thus all patients had at least two biliary lesions (strictures/irregularities) shown at endoscopic retrograde cholangiopancreatography. Patients with gall stones or established inflammatory bowel disease were excluded.

All patients were investigated for bowel pathogens at the time of diagnosis of AIDS related sclerosing cholangitis. ${ }^{7}$ At least three stools were cultured for faecal pathogens and mycobacteria; concentrated specimens were examined by direct microscopy for ova, cysts, and parasites, and smears were stained by a modified Ziehl Neelsen method with concentration for cryptosporidium. Each patient had a sigmoidoscopy with rectal biopsy; specimens were fixed in buffered formol saline, and standard paraffin sections were examined after staining with haematoxylin and 
eosin, and Ziehl Neelsen staining. Liver biopsy was performed if the prothrombin time and general state of the patient allowed. When cytomegalovirus was suspected, the diagnosis was confirmed immunohistochemically. Ampullary biopsies were taken at endoscopic retrograde cholangiopancreatography and similarly processed. The fundi were examined (after mydriatics) by an experienced ophthalmoscopist for evidence of cytomegalovirus retinitis. All patients had standard haematological and biochemical tests, and an upper abdominal ultrasound scan.

\section{CASE CONTROL STUDY}

For each patient with AIDS related sclerosing cholangitis a control was selected from the contemporaneous unit data base. The following criteria were required for selection as a control: AIDS diagnosis during the same calendar year as the case (similar duration of HIV positivity in non-AIDS cases); age within one year of case; CD4 count within $10 \%$ of case; evidence for cytomegalovirus and cryptosporidial enteritis

Figure 1: Cholangiogram in AIDS related sclerosing cholangitis. matched to case; normal liver function tests; absence of upper abdominal pain.

\section{Results}

CLINICAL AND LABORATORY PARAMETERS AT DIAGNOSIS

Nineteen patients were homosexual/bisexual men and one was a male heterosexual whose route of infection is unknown. The median age was 33.5 years (range 27-50), and the patients had been HIV seropositive for a median of 22 months (range 2-72) before diagnosis of AIDS related sclerosing cholangitis. Eighteen of the patients had a previous AIDS defining diagnosis. All patients had abdominal pain and 11 had diarrhoea. Nineteen of the patients had been treated with zidovudine, and 17 were on the drug at the time of diagnosis; two patients were taking dideoxyinosine. Other medication included prophylaxis for pneumocystis pneumonia in 17, acyclovir for recurrent herpes infections in 11, and triazole antifungals for candida in 14. One patient was on maintenance foscarnet for cytomegalovirus retinitis.

General examination revealed evidence of substantial weight loss ( $>10 \%$ ideal weight $)$ in 18 , and four had cutaneous Kaposi's sarcoma. None had clinical or microbiological evidence of bacterial sepsis. Five patients had smooth firm hepatomegaly; none had palpable splenomegaly. Liver biopsy, performed in 10 patients, showed changes compatible with sclerosing cholangitis in non-AIDS patients in five cases.

Standard liver function tests revealed serum alkaline phosphatase concentrations at least twice the upper limit of normal in 13 subjects, but a raised serum bilirubin in only three. Liver function tests were normal in four. The median CD4 lymphocyte count was $0.024 \times 10^{9} / 1$ (range $0.005-0.341$ ) (normal $>0.500$ ).

A potential infective cause for the condition was found in 15. Cryptosporidiosis was found in 13 (on ampullary biopsy in two, intestinal biopsy in five, and in the stools in 12). Cytomegalovirus infection was found contemporaneously at some site in the body in six individuals (ampullary biopsy in three, intestinal biopsy in five, and in the retina of one patient). Four patients had both infections. Cytology of smears obtained after insertion of a cytology brush into the biliary tree produced necrotic material only in five patients and cryptosporidial organisms were not isolated from bile samples of eight patients.

Upper abdominal ultrasound was abnormal in 10 ; the most common abnormality was of dilatation of the common bile duct (eight) but pathological biliary thickening was noted in three of these and in an additional two without duct dilatation.

All patients had many lesions on endoscopic retrograde cholangiography, which were confined to the intrahepatic system (above the confluence of the main right and left hepatic ducts) in two, limited to the extrahepatic ducts in three, and were more widespread in the remaining 15 (Fig 1). Two patients had apparently cystic lesions affecting the bile ducts (Fig 2). The pancreatic duct was markedly dilated in three 


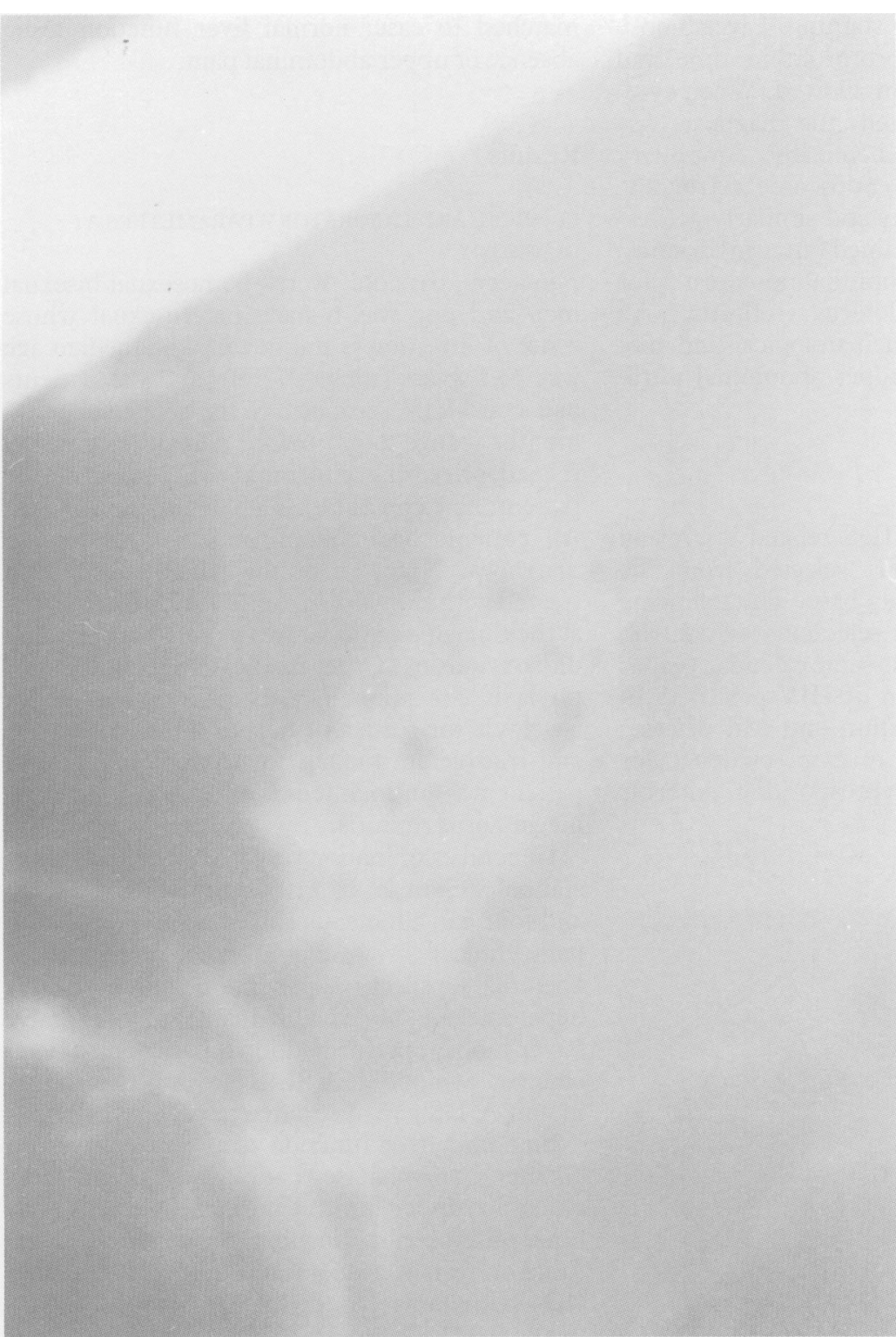

Figure 2: Cholangiogram in AIDS related sclerosing cholangitis showing apparent cysts affecting lower part of common bile duct (magnified view).

patients and in four individuals the duct exhibited minor changes similar to those seen in the elderly unaffected by HIV.

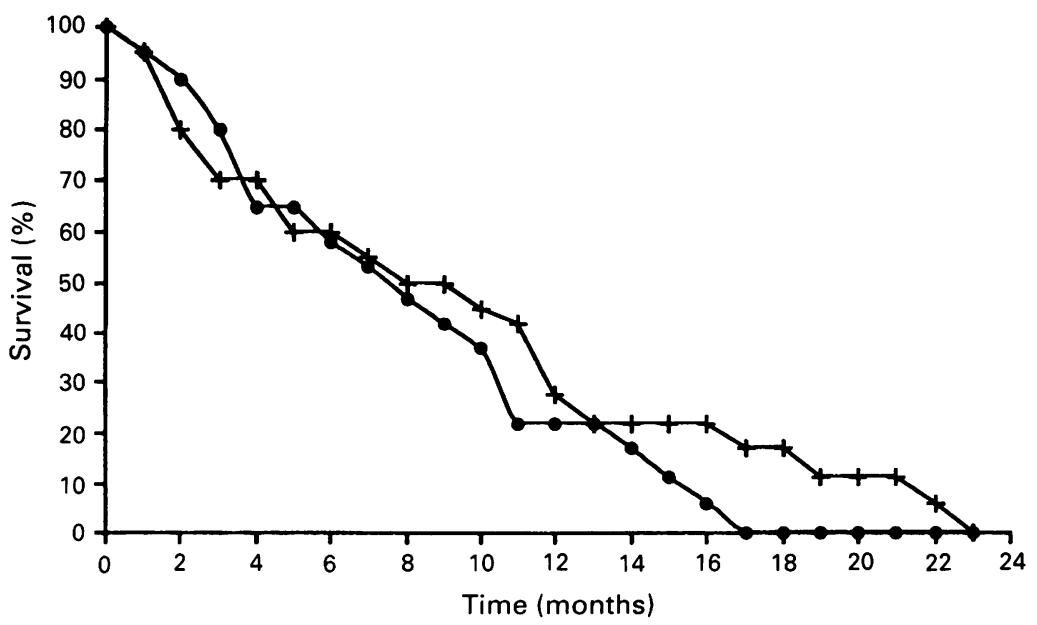

Figure 3: Kaplan-Meier survival plot (in months) for patients with AIDS related sclerosing cholangitis $(+)$ and for matched controls $(\mathrm{O})$

\section{IMMEDIATE MANAGEMENT}

All six patients with active cytomegalovirus infection were treated with infusions of foscarnet. In one, experimental therapy with interleukin II was attempted without success. In one patient in whom there appeared to be delay in biliary drainage at endoscopic retrograde cholangiopancreatography ( $>30$ minutes), an endoscopic sphincterotomy was performed. No attempt at specific therapy was made in the remaining 12 patients. Analgesics were given in increasing doses and ascending potency until pain was controlled. Opiates (usually morphine) were required at least temporarily in all. Diarrhoea was treated symptomatically, usually coincidentally by morphine given primarily for pain.

\section{PROGRESS AND PROGNOSIS}

Seventeen patients have died, at a median of seven months from the time of diagnosis of AIDS related sclerosing cholangitis (range 1-23 months). Three patients are alive and free of pain at 10,11 , and 21 months after (Fig 3).

In four patients abdominal pain, controlled by analgesics, persisted until death at $2,2,8$, and 12 months from the time of diagnosis. In one patient symptoms became much less marked after one month but persisted to death at three months. The patient in whom sphincterotomy was performed had a reduced analgesic requirement for three months but subsequently relapsed; pain control remained a problem to death at 19 months, with progression of the cholangiographic abnormalities shown at 15 months (Fig 4). In the remaining 14 patients, symptoms resolved (apparently spontaneously) after a median of four weeks (range 2-26 weeks): 11 of these have died from apparently unrelated causes without relapse of symptoms attributable to AIDS related sclerosing cholangitis.

The median time to symptom control in those with cytomegalovirus infection (and therefore treated with foscarnet) was four weeks, which does not differ from the median time to control on those without cytomegalovirus infection treated with analgesics alone. One of the patients with uncontrolled pain had active cytomegalovirus. The median survival of $7 \cdot 5$ months (range 2-13) for patients with cytomegalovirus and AIDS related sclerosing cholangitis is identical to that for the whole AIDS related sclerosing cholangitis group. Neither the presence of cryptosporidiosis nor the absence of enteric pathogens had a significant effect on prognosis.

In 13 cases liver function tests improved or remained static from the time of diagnosis, and have remained normal in two of those still alive who had normal values at diagnosis. In seven patients (including one with initially normal results), however, alkaline phosphatase and bilirubin have risen since diagnosis; six of these are dead. Initial alkaline phosphatase concentration showed a weak inverse correlation with survival $(r=-0.3)$ which did not reach statistical significance; a rising titre after diagnosis may predict a poorer prognosis but numbers are small.

Increasing age was significantly protective in 

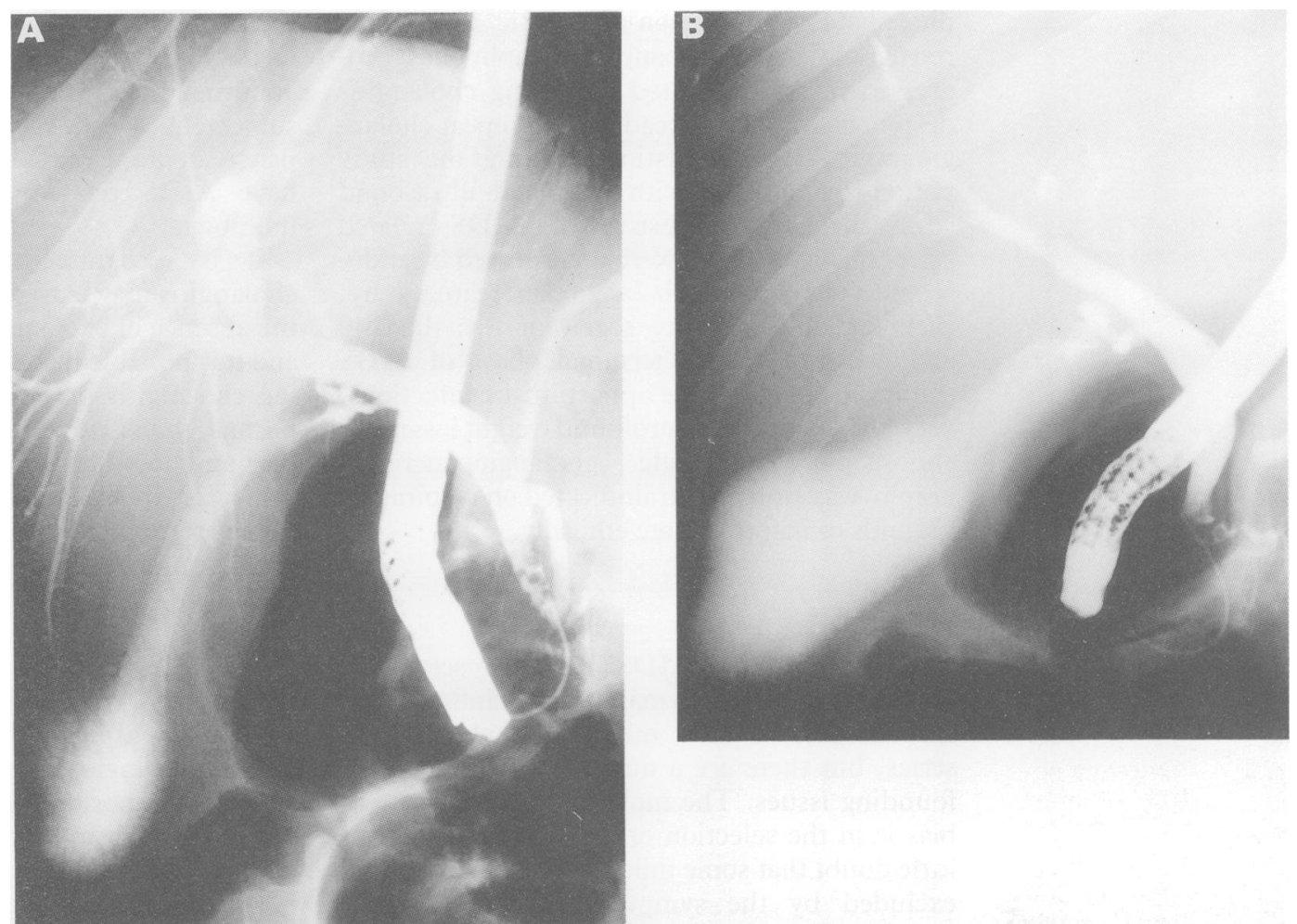

Figure 4: Progression of cholangiographic extent of AIDS related sclerosing cholangitis in patient with uncontrolled symptoms: at diagnosis (left); at 15 months follow up (right) - introduction of further contrast above an occluding balloon permitted no additional intrahepatic filling.

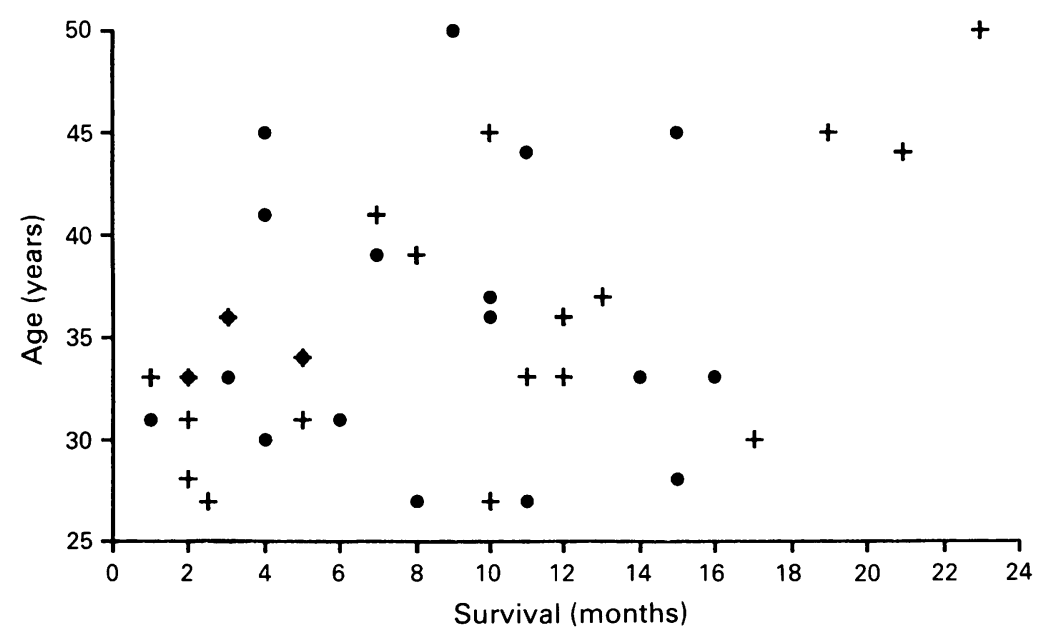

Figure 5: Effect of age on prognosis (in months) for patients $(+)$ and controls $(O)$.

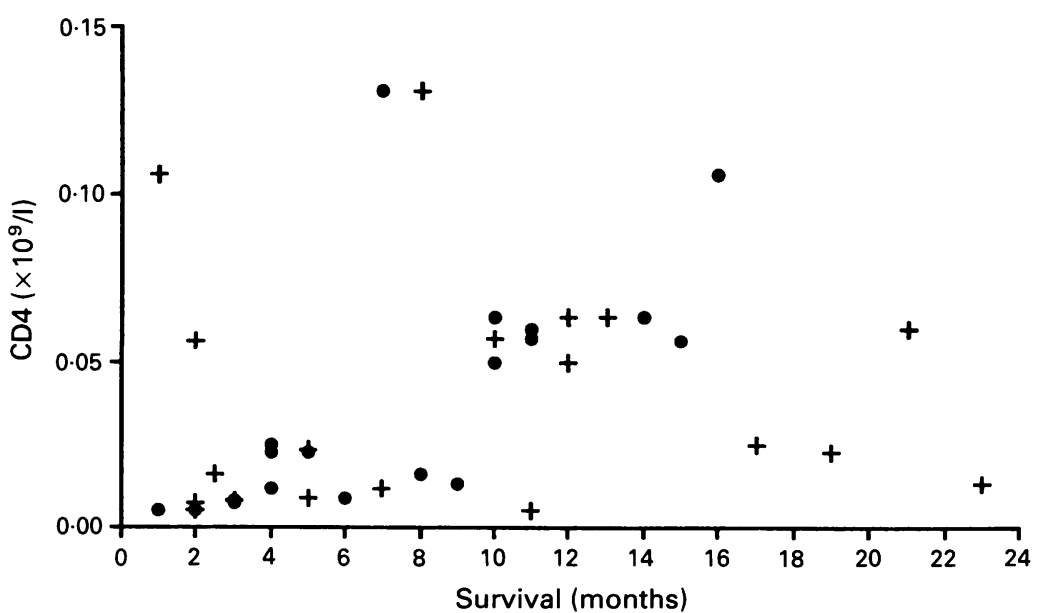

Figure 6: Relation of CD4 count $\left(\times 10^{9} / l\right)$ to prognosis in patients $(+)$ and controls $(O)$. The patient and control with CD4 of 0.34 had survivals of 10 and 15 months respectively (not illustrated).
AIDS related sclerosing cholangitis $(r=0 \cdot 64)$ (Fig 5). Median survival in patients below the median age for the present group was only 3.5 months as compared with 11 months in those above the median age $(\mathrm{p}<0.05 ; 2$-tailed MannWhitney).

CD4 count at AIDS related sclerosing cholangitis diagnosis did not correlate with survival $(r=0 \cdot 1)$ (Fig 6). Although all those dying within six months of diagnosis had an initial CD 4 of less than $0 \cdot 11 \times 10^{9} / 1$, three patients with CD 4 counts below 0.05 have survived for 17 months or more.

\section{CASE CONTROL STUDY}

Despite the stringent criteria it was possible to identify a matched control for each case. Survival of the controls was to a median of 7.5 months (range 1 to $>16$ ) (Fig 3) which corresponds remarkably closely to that in cases $(7 \cdot 5 ; 1-23)$. The controls showed a weak negative correlation between age and survival (Fig 5), and a strong positive correlation between CD4 count and survival $(r=0.60 ; p<0.05)$ (Fig 6).

The overall incidence of opportunistic enteritis within the unit during the period of recruitment of AIDS related sclerosing cholangitis patients included 82 cases of chronic cryptosporidiosis, 49 cases of cytomegalovirus colitis (in isolation), and 18 cases of cytomegalovirus enteritis at other or multiple sites. During the study period, five additional patients had endoscopic retrograde cholangiopancreatography for putative AIDS related sclerosing cholangitis; in two cases there were intraductal stones, and in the remainder no definite abnormality was identified. To date no patient with right upper quadrant pain and a raised alkaline 
phosphatase but a non-diagnostic endoscopic retrograde cholangiopancreatography has progressed to AIDS related sclerosing cholangitis despite an increasing readiness to repeat cholangiograms in this situation. During the study period six patients with pain and ultrasound scans strongly suggestive of AIDS related sclerosing cholangitis were not assessed by endoscopic retrograde cholangiopancreatography; each had a Karnofsky score $^{8}$ of less than 40 and had entered the terminal phase of AIDS (multiple unresponsive opportunistic infections, very low CD4 counts, profound weight loss), and endoscopic retrograde cholangiopancreatography was either contraindicated on respiratory grounds or inappropriate ethically.

\section{Discussion}

It appears that AIDS related sclerosing cholangitis had had no major overall influence on the outcome of HIV infection in the present series, but there are a number of possible confounding issues. The most important source of bias is in the selection of patients, and there is little doubt that some mild/early cases have been excluded by the symptom led investigatory protocol and by the requirement for unequivocal features at endoscopic retrograde cholangiopancreatography. On the other hand patients presenting with very advanced disease were not included through compassionate avoidance of endoscopic retrograde cholangiopancreatography.

That AIDS related sclerosing cholangitis is the cause of pain in affected patients is supported by earlier work in HIV-infected patients ${ }^{5}$ (which included 10 of the patients described here) where a diagnosis of AIDS related sclerosing cholangitis was only made after the exclusion of other causes of pain, but the possibility that pain arises in the pancreas is raised by the discovery of coexistent abnormal pancreatograms. A primarily pancreatic origin is, however, unlikely given the absence of typical pancreatic pain, or pancreatic malabsorption, the presence of a normal pancreatogram in the majority of AIDS related sclerosing cholangitis patients and a lack of pancreatic abnormalities on ultrasound or computed tomography scan. Moreover there were no clinical or prognostic differences between those with and those without abnormal pancreatic radiology. It seems likely that the abnormalities in both biliary and pancreatic ducts are different manifestations of the AIDS related sclerosing cholangitis syndrome. Apparently cystic abnormalities within the biliary ducts of several of the present patients do not appear to have been recognised in other contexts: it is suggested that these too are a constituent of the radiology of AIDS related sclerosing cholangitis.

Unlike Payne et $a l,{ }^{9}$ we have found a high frequency of AIDS related sclerosing cholangitis in AIDS patients with substantially raised serum alkaline phosphatase. This may well reflect differences in case definition and the limitations inherent in their retrospective study, in which endoscopic retrograde cholangiopancreatography was not a routine investigation. Never- theless there are, in both units, large cohorts of patients with HIV infection with mildly abnormal liver function tests who have not been subjected to endoscopic retrograde cholangiopancreatography. Whether these individuals have AIDS related sclerosing cholangitis is unknown. ${ }^{10}$

It is recognised that primary sclerosing cholangitis may exist (on histological criteria) in the absence of cholangiographic abnormalities," and it is possible that early AIDS related sclerosing cholangitis may likewise be present with a normal cholangiogram. Although patients with pain and obstructive liver function tests are now subjected to liver biopsy after negative endoscopic retrograde cholangiopancreatography, a consistent histological pattern is yet to be recognised.

\section{AETIOLOGY}

The threshold for performing endoscopic retrograde cholangiopancreatography on patients with cryptosporidial infection is low given the suggestion that many (or even all) patients with cryptosporidiosis have biliary involvement. ${ }^{+}$ It appears that about two-thirds of well documented cases of AIDS related sclerosing cholangitis in the United Kingdom will prove infected, and that at least a sixth of patients with cryptosporidiosis will have AIDS related sclerosing cholangitis if subjected to endoscopic retrograde cholangiopancreatography. Our policy on endoscopic retrograde cholangiopancreatography may have led us to over estimate the association, but without performing endoscopic retrograde cholangiopancreatography on unselected patients with HIV infection (with and without cryptosporidiosis), precise figures cannot be given.

Cytomegalovirus was associated with AIDS related sclerosing cholangitis in only two of the present patients in whom cryptosporidiosis was not also implicated and in less than a third overall; these represent less than $10 \%$ of all cases of cytomegalovirus enteritis seen during the study period. The early impression that the virus was of prime aetiological significance in AIDS related sclerosing cholangitis ${ }^{1}$ is not supported by the present prevalence; the absence of a better outlook in AIDS related sclerosing cholangitis patients (with cytomegalovirus) treated with foscarnet compared with untreated patients is consistent with the conclusion that it is unlikely to be important.

A quarter of the present patients had no identifiable gastrointestinal infection/infestation; it is possible that new organisms or occult infection are implicated, and, although unlikely given the absence of CD4 receptors on healthy biliary epithelium, ${ }^{12} \mathrm{HIV}$ may itself damage the biliary tree. An autoimmune aetiology for AIDS related sclerosing cholangitis akin to that of primary sclerosing cholangitis is also possible. Although autoimmune phenomena in HIV disease seem inherently unlikely, there is increasing evidence that these occur and may be important in pathogenesis. ${ }^{13}$

As inflammatory bowel disease occurs with approximately equal frequency in the HIV sero- 
positive and control populations, ${ }^{5}$ it must be assumed that primary sclerosing cholangitis may also, and a clear distinction between AIDS related sclerosing cholangitis and primary sclerosing cholangitis would not then be possible; this has not yet been a diagnostic problem. Although a small proportion of patients with primary sclerosing cholangitis develop colitis only after the biliary presentation ${ }^{11}$ - follow up of AIDS related sclerosing cholangitis patients (particularly those with pathogen negative diarrhoea) has not yet yielded any patients with noninfective colitis.

\section{PROGRESS AND PROGNOSIS}

In primary sclerosing cholangitis standard liver function tests may improve after diagnosis ${ }^{14}-\mathrm{a}$ result of diagnosis tending to be at times of maximal abnormality in a fluctuating course and likely therefore to be followed by a period of partial remission. A similar phenomenon has been observed in some patients with AIDS related sclerosing cholangitis, but a halting progression with worsening of the biliary disease is not often seen, and there are no reports of hepatic fibrosis deteriorating to cirrhosis and liver failure. Such progression may of course merely be preempted by the worse prognosis of HIV disease itself. The present data indicate that a suggested prognostic scoring system for primary sclerosing cholangitis ${ }^{15}$ based on the presence or absence of hepatomegaly and splenomegaly, the serum alkaline phosphatase, the patient's age, and the histological stage is not valid in AIDS related sclerosing cholangitis.

Presently available therapeutic interventions appear without value in AIDS related sclerosing cholangitis. When the urgently needed effective treatment for cryptosporidiosis emerges it will be important to assess its effect in AIDS related sclerosing cholangitis, not least because indirect information on the pathogenesis of the condition should result. Although occasional patients with evidence of biliary outflow obstruction due to ampullary stenosis have been described, ${ }^{10}$ we saw only one patient in this study with delayed biliary drainage. Although anecdotally, sphincterotomy has been suggested to improve pain, it is difficult currently to justify such an approach outside the context of controlled trials, particularly as spontaneous resolution of pain occurred in a number of our patients.

In contrast with patients with primary sclerosing cholangitis, ${ }^{15}$ and to $\mathrm{HIV}$-infected patients in general, ${ }^{16}$ increasing age was protective in AIDS related sclerosing cholangitis; this paradoxical effect is unexplained but has wide confidence limits and a type I error (of small numbers) cannot be absolutely excluded. The effects of differential immunosuppression and of coexistent serious opportunistic infections have probably been important given the poor protection effected by youth in our matched controls, but the significant reversal of even this minor association should probably not be ignored. This is especially relevant since the presence of cytomegalovirus and/or cryptosporidiosis had little prognostic significance, and CD4 levels correlated poorly with prognosis. It may not be coincidental that two of the long survivors with very low $\mathrm{CD} 4$ counts were also the oldest patients in the study, and it remains possible that the genetic background or immune changes responsible for AIDS related sclerosing cholangitis are, with increasing age, more likely to indicate a good prognosis independently of the CD4 count.

Part of this work was presented at the meeting of the British Society of Gastroenterology in London, September 1991. We thank Dr I M Murray-Lyon and Dr M G Anderson for allowing us to report on patients under their care, Dr G M Connolly who played a major role in data collection, and $\mathrm{Mr} \mathrm{J}$ Perrett who identified several of the control patients.

1 Cello JP. Acquired immunodeficiency syndrome cholangiopathy: spectrum of disease. Am F Med 1989; 86: 539-46.

2 Dowsett JF, Miller R, Davidson R, et al. Sclerosing cholangitis in acquired immunodeficiency syndrome. Scand f Gastroenterol 1988; 23: 1267-74.

3 Yana C, Frija J, Cyna-Gorse F, et al. Aspects radiologiques des inflammations non calculeuses des voies biliaires au cours du inflammations non calculeuses des
SIDA. F Radiol $1989 ; 70: 411-4$.

4 Gross TL, Wheat J, Bartlett M, O'Connor KW. AIDS and multiple system involvement with cryptosporidium. $A m \mathcal{J}$ Gastroenterol 1986; 81: 456-8.

5 Thuluvath PJ, Connolly GM, Forbes A, Gazzard BG Abdominal pain in HIV infection. $Q \mathcal{F}$ Med 1991; 78: 275-

6 Chapman RWG, Arborgh BAM, Rhodes JM, Summerfield JA, Dick R, Scheuer PJ, et al. Primary sclerosing cholangitis: a review of its clinical features, cholangiography and hepatic histology. Gut 1980;21: 870-7.

7 Connolly GM, Forbes A, Gazzard BG. Investigation of seemingly pathogen-negative diarrhoea in patients infected seemingly pathogen-negative dia
with HIVl. Gut 1990; 31: 886-9.

8 Karnofsky DA, Abelmann WH, Craven LF, Burchenall JH. The use of nitrogen mustards in palliative treatment of The use of nitrogen mustards in

9 Payne TH, Cohn DL, Davidson AJ, Henry TD, Schaefer JW, Gabow PA. Marked elevations of serum alkaline phosphatase in patients with AIDS. Fournal of Acquired Immun Deficiency Syndromes 1991; 4: 238-43.

10 Schneiderman DJ, Cello JP, Laing FC. Papillary stenosis and sclerosing cholangitis in the acquired immunodeficiency syndrome. Ann Intern Med 1987; 106: 546-9.

11 Chapman RW. Aetiology and natural history of primary sclerosing cholangitis - a decade of progress? Gut 1991; 32 . 1433-5.

12 Hata K, Zhang XR, Iwatsuki S, Van Thiel DH, Herberman RB, Whiteside TL. Isolation, phenotyping, and functional analysis of lymphocytes from human liver. Clin Immunol Immunopathol 1990; 56: 401-19.

13 Maddox J. AIDS research turned upside down. Nature 1991; 353: 297.

14 Holdstock G, Millward-Sadler GH, Wright R. Hepatic changes in systemic disease. In: Wright R, Millward-Sadler $\mathrm{GH}$, Alberti KGMM, Karran S, eds. Liver and biliar disease. 2nd ed. London: Bailliere Tindall, 1985: 1033-76.

15 Farrant JM, Hayllar KM, Wilkinson ML, et al. Natura history and prognostic variables in primary sclerosing cholangitis. Gastroenterology 1991; 100: 1710-7.

16 Moore RD, Hidalgo J, Sugland BW, Chaisson RE Zidovudine and the natural history of the acquired immunodeficiency syndrome. N Engl f Med 1991; 324: 1412-5. 